\title{
Numerical Modeling and Control of Rotating Plate with Coupled Self-Sensing and Frequency-Dependent Active Constrained Layer Damping
}

\author{
Zhengchao Xie, ${ }^{1}$ Pak Kin Wong, ${ }^{1}$ Long Zhang, ${ }^{2}$ \\ and Hang Cheong Wong ${ }^{1}$ \\ ${ }^{1}$ Department of Electromechanical Engineering, University of Macau, Macau, China \\ ${ }^{2}$ Magnecomp Precision Technology, 38975 Skycanyon Drive, Murrieta, CA 92563, USA \\ Correspondence should be addressed to Zhengchao Xie, zxie@umac.mo
}

Received 31 May 2012; Revised 14 September 2012; Accepted 5 October 2012

Academic Editor: Slim Choura

Copyright (C) 2012 Zhengchao Xie et al. This is an open access article distributed under the Creative Commons Attribution License, which permits unrestricted use, distribution, and reproduction in any medium, provided the original work is properly cited.

This work proposes a coupled finite element model for actively controlled constrained layer damped (CLD) rotating plate with self-sensing technique and frequency-dependent material property in both the time and frequency domain analyses. Constrained layer damping with viscoelastic material can effectively reduce the vibration in rotating structures. However, most existing research models use complex modulus approach to model the viscoelastic material, but it limits to frequency domain analysis and the frequency dependency of the viscoelastic material is not well-included as well. It is meaningful use of the anelastic displacement fields (ADFs) that is done in order to include the frequency dependency of the material for both the time and frequency domains. Also, unlike previous ones, all types of damping are taken into account by this finite element model. Thus, in this work, a single layer finite element is adopted to model a threelayer active constrained layer damped rotating plate in which the constraining layer is made of piezoelectric material to work as both the self-sensing sensor and actuator. This newly proposed finite element model is validated, and then, as shown in numerical studies, this proposed approach can achieve effective vibration reduction in both the frequency and time domains.

\section{Introduction}

Constrained layer damping (CLD) is an effective vibration reduction approach in which a damping layer made of viscoelastic material is sandwiched between the base structure and a constraining layer. In the deformation of this damping layer, a portion of the strain energy of the viscoelastic material is dissipated so that vibrational damping can be achieved. The 


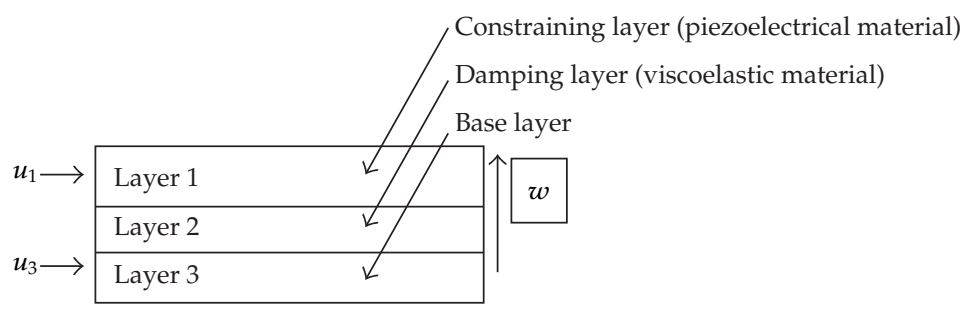

Figure 1: RUK model displacements for a CLD sandwich beam.

damping ratio achieved by using this viscoelastic material is dependent on the frequency and environment temperature. At present, a wide variety of viscoelastic materials exists, so the study on the damping property of the viscoelastic material is an interesting topic.

Recently, constrained layer damping is employed for the vibration suppressions of rotating structures, which has wide applications in industries such as wind turbines and helicopters. Rotating beams, as simplification of rotating plates, have been extensively investigated, including fundamental dynamics [1]. Yoo and Pierre [2] studied the dynamics of rotating bare plates using analytical approach. Based on the work in [2], Liu and Hong [3] developed a plate finite element model for a rotating bare plate. Xie and Xue [4] proposed a finite element model on passive constrained layer damped plate which can include more types of damping in the structure and a parametric study was conduced in order to check the performance of different constrained layer damping configurations. Fung and Yau [5] studied Active CLD (ACLD) application on rotating beam. Liu et al. [6] extended their own work in [3] to active control of constrained layer damped rotating plates through finite element approach. In ACLD technique, a voltage is applied on the piezoelectric constraining layer to generate active force. Dosch et al. [7] proposed self-sensing technique that piezoelectric material can be used as both the actuator and sensor. Based on the work of [7], Gao and Liao [8] and Shen [9] implemented ACLD rotating beam where the piezoelectric constraining layer works as both the sensor and actuator, and a bridge circuit [7] is used to extract the sensed voltage from measured signal. One of advantages for this approach is that it can achieve the true collocated control which means the control systems is unconditionally stable [10]. Xie et al [11] proposed a proportional control on ACLD rotating plate and an optimization based on genetic algorithms, and the newly developed dynamic model of the present work is partially based on [11]. Similar to the work in [4], a parametric study was conducted in [11] to check the impact of different active CLD configurations on the vibration reduction of rotating plates in the frequency domain.

It is worth noting that all of the work mentioned above utilized the same assumptions as those in RKU model [12] that only shear strain exists in the core layer and extension strain in the constraining and base layers. In this assumption, the two face layers are treated as Euler-Beams while the damping layer only experiences shear deformation. The displacements associated with this assumption are illustrated in Figure 1. For the convenience of the discussion, two face layers are called the constraining layer and the base layer respectively in this paper, while the middle layer is called the damping layer or core layer. The layers are also denoted by numbers 1, 2 and 3, respectively, throughout this work. The displacements $u_{1}$ and $u_{3}$ are employed to represent the longitudinal displacements of face layers 1 and 2, respectively. $w$ is utilized to denote the transverse displacement of sandwich structure, which is identical for each of the three layers. Shear deformation in the core layer 
is determined from the relative motion between $u_{1}$ and $u_{3}$. While the assumptions described above are widely used, they are not always valid in certain structures when RKU model is employed. Rao [13] studied the problem using the same transverse displacement assumption as that in [12] but with additional assumptions that the longitudinal displacement varies linearly across the thickness of each layer, and the displacement is continuously across the layer connections. In that work, all of three layers are treated as Timoshenko beams instead of Euler beams used in the RKU model. So, all three layers can have both the shear and normal strains and so this could better predict the damping in the structure compared with assumptions in RKU model. Zapfe and Lesieutre [14] presented a beam finite element that can be regarded as the finite element implementation of the Rao's model.

As mentioned previously, viscoelastic material in CLD structures should have frequency dependent property, and currently most existing research assumes the complex modulus for viscoelastic materials has included the frequency dependency of the material. The limitation for this complex modulus approach is that the model can only be studied in the frequency domain. There are some other independent approaches to investigate this frequency dependency in time domain. Trindade et al. [15] gave a review on several approaches such as anelastic displacement fields (ADF), Golla-Hughes-McTavish (GHM), and iterative modal strain energy (MSE) method on modeling the frequency dependency of viscoelastic material in the composite beams with a viscoelastic damping layer. The ADF and GHM approach have been proven to be effective in studying the constrained layer damping in the time domain, and the ADF approach is better than GHM on reducing the model size and providing more accurate prediction $[15,16]$. So, in this work ADF is employed to model the frequency dependency of viscoelastic material in the damping layer.

In this research, a plate finite element is developed to model the multilayer rotating plates. This finite element plate model extends previous research from beam to plate, and it is expected that more possible types of damping can be captured for practical rotating plate analysis due to the proposed displacement field. This feature overcomes the oversimplification in previous works. Also, this finite element plate model can be extended to multilayer (more than three) rotating plates conveniently. Then, active vibration control is implemented with a self-sensing constraining layer, which means the piezoelectric constraining layer works as both the sensor and the actuator. ADF approach is also adopted to include the frequency dependency of viscoelastic material of the damping layer to make

it possible to analyze the structure in both the time and frequency domains. Then, the newly proposed model is validated. At last, a numerical study is conducted to investigate the performance of this new numerical model.

\section{Finite Element Modeling}

\subsection{Assumptions and Kinematics Relations}

Prior to introducing the rotating plate finite element model, the assumptions as those in [11] should be introduced. Note that the assumptions in [13] are for beams.

(1) The transverse displacements of all three layers are equal.

(2) The longitudinal displacement is linearly distributed across the thickness of each layer.

(3) There is no slip between the layers. 

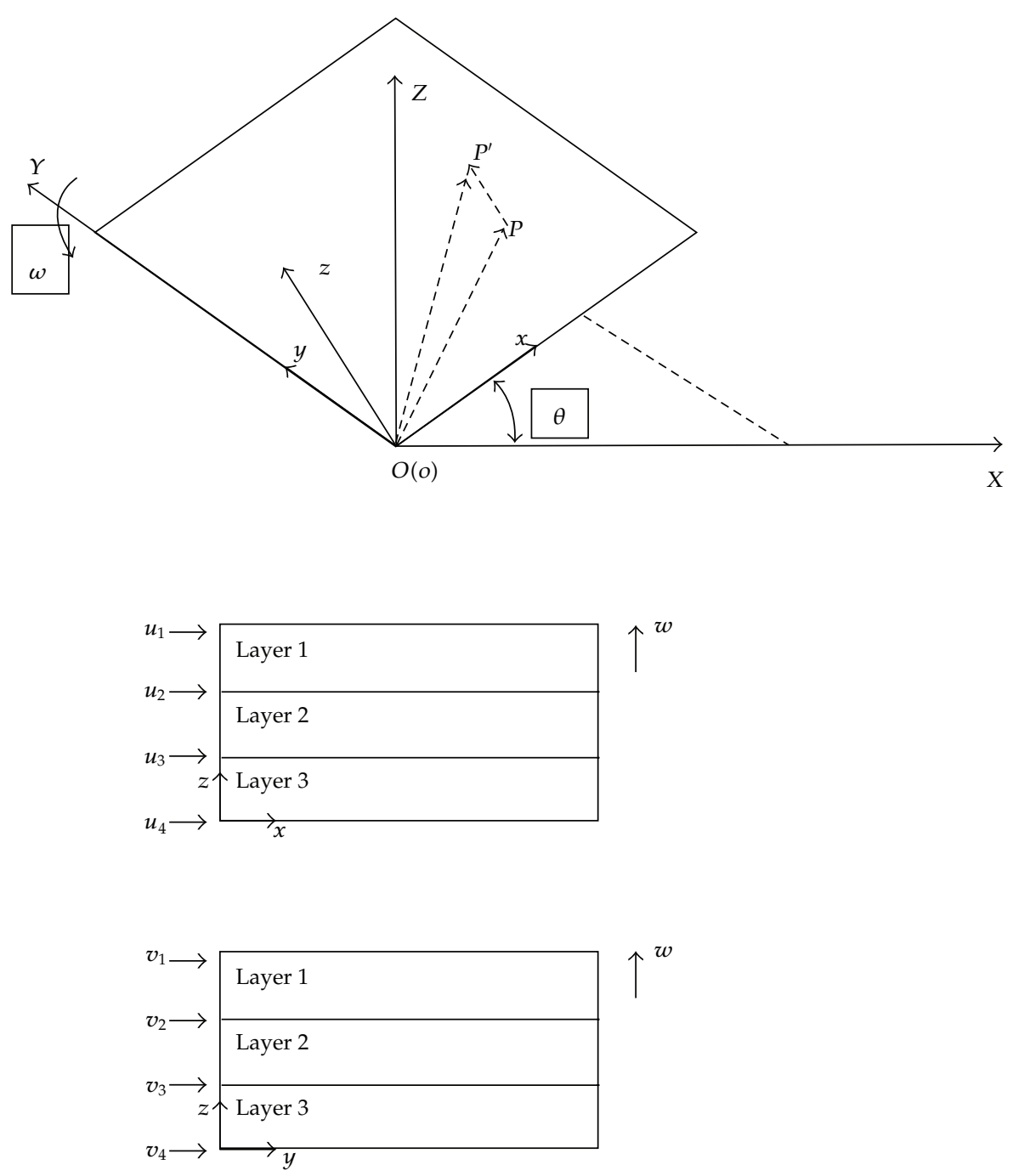

Figure 2: Proposed displacement field [11] (direction of angular velocity is set as along the $-Y$ axis).

The rotating plate is modeled in this work based on above assumptions. Figure 2 shows the proposed displacement field for the rotating plate with angular velocity $\omega . w$ is the transverse displacement for all of the three layers. $u_{i}$ and $v_{i}$ are the longitudinal displacements at the contact surfaces of different layers along the $x$ - and $y$-axes, respectively. The longitudinal displacements of each layer vary linearly across the thickness of each layer. Note that there are two coordinate systems in Figure 2, a rotating coordinate system o- $x y z$ attached to the rotating plate, and a global coordinate system $O-X Y Z$ that is fixed without rotation. The transformation matrix between two coordinate systems is defined as in [3]:

$$
A=\left[\begin{array}{ccc}
\cos \theta & 0 & -\sin \theta \\
0 & 1 & 0 \\
\sin \theta & 0 & \cos \theta
\end{array}\right] .
$$


Taking an arbitrary point on the rotating plate which moves from $P$ to $P^{\prime}$ after the deformation, the $O P_{x y z}^{\prime}=\left[\begin{array}{l}x \\ y \\ 0\end{array}\right]+\left[\begin{array}{c}u \\ w \\ w\end{array}\right]+\left[\begin{array}{c}-\int_{0}^{x}(\partial w / \partial x)^{2} d x \\ -\int_{0}^{y}(\partial w / \partial y)^{2} d y \\ 0\end{array}\right]$. The term $\left[\begin{array}{c}-\int_{0}^{x}(\partial w / \partial x)^{2} d x \\ -\int_{0}^{y}(\partial w / \partial y)^{2} d y \\ 0\end{array}\right]$ is the so-called "stiffening effect" [3], which are the coupling terms between the transverse displacement and the longitudinal displacement due to the centrifugal force of the rotation. The transformation of the displacement from the $o-x y z$ coordinate system to $O-X Y Z$ coordinate system can be made as follows:

$$
O P_{X Y Z}^{\prime}=A\left(\left[\begin{array}{l}
x \\
y \\
0
\end{array}\right]+\left[\begin{array}{c}
u \\
v \\
w
\end{array}\right]+\left[\begin{array}{c}
-\int_{0}^{x}\left(\frac{\partial w}{\partial x}\right)^{2} d x \\
-\int_{0}^{y}\left(\frac{\partial w}{\partial y}\right)^{2} d y \\
0
\end{array}\right]\right)
$$

With the proposed displacement field, kinetic and strain energy can be obtained respectively, based on that, the equations of motion can be derived.

\subsection{Strain and Kinetic Energy}

Given displacement field, the elastic strain energy of the $i$ th layer in the rotating plate can be represented as:

$$
\begin{aligned}
V_{i}=\frac{1}{6} \iiint_{V_{i}}\left(E_{i}\right. & \left(\left(\frac{\partial u_{i}}{\partial x}\right)^{2}+\frac{\partial u_{i}}{\partial x} \frac{\partial u_{i+1}}{\partial x}+\left(\frac{\partial u_{i+1}}{\partial x}\right)^{2}\right) \\
& +3 G_{i}\left(\frac{\partial w_{i}}{\partial x}-\frac{u_{i}-u_{i+1}}{2 H_{i}}\right)^{2}+3 * E_{i} I_{i} \frac{\partial^{2} w}{\partial x^{2}} \\
& \left.+E_{i}\left(\left(\frac{\partial v_{i}}{\partial x}\right)^{2}+\frac{\partial v_{i}}{\partial x} \frac{\partial v_{i+1}}{\partial x}+\left(\frac{\partial v_{i+1}}{\partial x}\right)^{2}\right)+3 G_{i}\left(\frac{\partial w_{i}}{\partial x}-\frac{v_{i}-v_{i+1}}{2 H_{i}}\right)^{2}\right) d v
\end{aligned}
$$

where $H_{i}$ is the thickness of the $i$ th layer.

The kinetic energy is dependent on the motion of the structure. Taking the time derivative of displacement $O P_{X Y Z}^{\prime}$ in (2.3) gives:

$$
O \dot{P}_{X Y Z}^{\prime}=\dot{A}\left[\begin{array}{c}
x+u-\int_{0}^{x}\left(\frac{\partial w}{\partial x}\right)^{2} d x \\
y+v-\int_{0}^{y}\left(\frac{\partial w}{\partial y}\right)^{2} d y
\end{array}\right]+A\left[\begin{array}{c}
\dot{u}-\frac{\partial}{\partial t}\left(\int_{0}^{x}\left(\frac{\partial w}{\partial x}\right)^{2} d x\right) \\
\dot{v}-\frac{\partial}{\partial t}\left(\int_{0}^{y}\left(\frac{\partial w}{\partial y}\right)^{2} d y\right)
\end{array}\right] .
$$



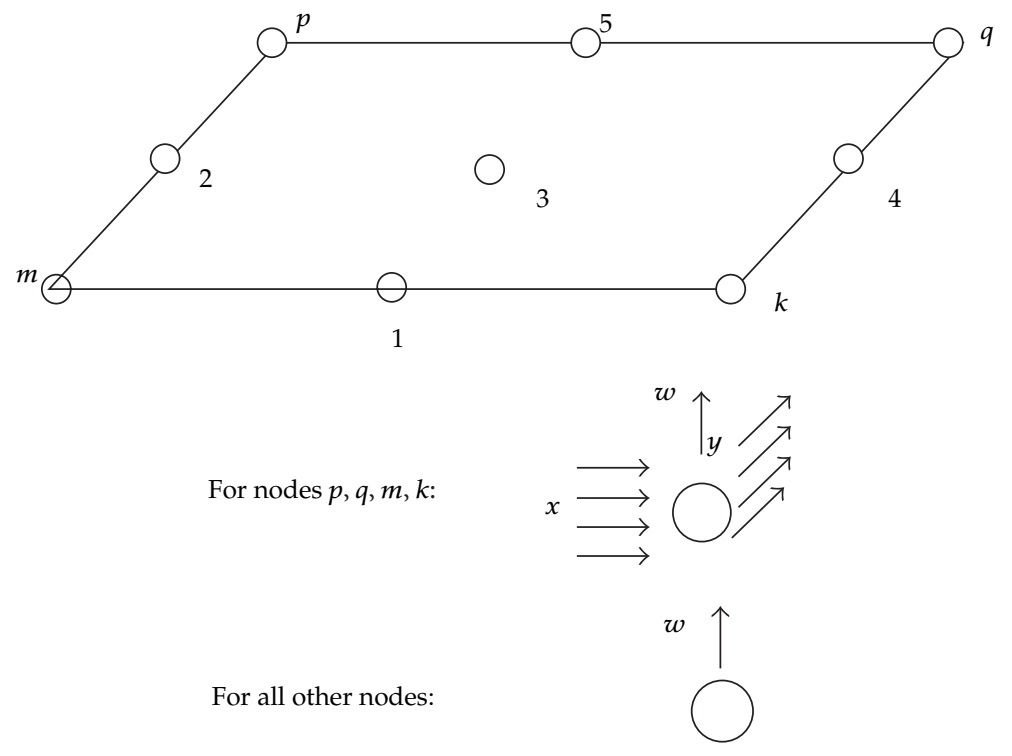

Figure 3: Proposed new plate finite element [11].

The kinetic energy of the $i$ th layer then can be calculated as:

$$
\begin{array}{r}
T_{i}=\frac{1}{2} \rho_{i} \iiint_{V_{i}}\left(\left(\dot{u}_{i}^{2}+\dot{v}_{i}^{2}+\dot{w}_{i}^{2}\right)+w\left(2 x \dot{w}+\dot{w} u_{i}+u_{i} \dot{w}-\dot{u}_{i} w-w \dot{u}_{i}\right)\right. \\
\left.+\omega^{2}\left(x^{2}+u_{i}^{2}-x \int_{0}^{x}\left(\frac{\partial w}{\partial x}\right)^{2} d x+2 x u_{i}+w^{2}\right)\right) d v
\end{array}
$$

where $\omega=\dot{\theta}$ is the angular velocity of plate rotation.

\subsection{Finite Element Discretization}

As mentioned earlier, once the kinetic and the strain energy are obtained, the displacement field can be discretized using the new plate finite element. Figure 3 shows the $i$ th layer of the proposed new plate finite element. Here it is worth noting that the mid nodes are employed to avoid shear locking [13]. As a result, there are 25 degrees of freedom for the $i$ th layer as shown in Figure 3 and 41 degrees of freedom for all of three layers.

The displacement vector of the $i$ th layer with this new plate finite element becomes as follows:

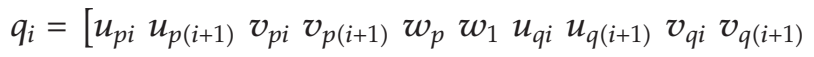

$$
\begin{aligned}
& \left.\begin{array}{lllllllllllllll}
w_{q} & w_{2} & w_{3} & w_{4} & u_{m i} & u_{m(i+1)} & v_{m i} & v_{m(i+1)} & w_{m} & w_{5} & u_{k i} & u_{k i} & v_{k i} & v_{k(i+1)} & w_{k}
\end{array}\right]^{T} .
\end{aligned}
$$


For the $i$ th layer of plate finite element, the proposed displacement field (Figure 3) can be organized as:

$$
\left[\begin{array}{c}
u \\
v \\
w
\end{array}\right]_{i}=B\left[\begin{array}{c}
u_{i} \\
u_{i+1} \\
v_{i} \\
v_{i+1} \\
w
\end{array}\right]=B\left[\begin{array}{l}
F_{1} \\
F_{2} \\
F_{3} \\
F_{4} \\
F_{5}
\end{array}\right] q_{i}=B F q_{i}=N q_{i}
$$

and the dimensions of matrix $B$ and $F$ are $3 \times 5$ and $5 \times 25$, respectively.

Substituting Equation (2.7) into (2.2), (2.3) and (2.5), and neglecting the high order terms, the kinetic and strain energy of the $i$ th flayer can be reorganized as:

$$
\begin{gathered}
V_{i}=\frac{1}{6} \iiint_{V_{i}}\left(E_{i} q_{i}^{T}\left(\left(\frac{\partial F_{1}}{\partial x}\right)^{T}\left(\frac{\partial F_{1}}{\partial x}\right)+\left(\frac{\partial F_{1}}{\partial x}\right)^{T}\left(\frac{\partial F_{2}}{\partial x}\right)+\left(\frac{\partial F_{2}}{\partial x}\right)^{T}\left(\frac{\partial F_{2}}{\partial x}\right)\right) q_{i}\right. \\
+E_{i} q_{i}^{T}\left(\left(\frac{\partial F_{3}}{\partial y}\right)^{T}\left(\frac{\partial F_{3}}{\partial y}\right)+\left(\frac{\partial F_{3}}{\partial y}\right)^{T}\left(\frac{\partial F_{4}}{\partial y}\right)+\left(\frac{\partial F_{4}}{\partial y}\right)^{T}\left(\frac{\partial F_{4}}{\partial y}\right)\right) q_{i} \\
+3 G_{i} q_{i}^{T}\left(\frac{\partial F_{5}}{\partial x}-\frac{F_{1}-F_{2}}{2 H_{i}}\right)^{T}\left(\frac{\partial F_{5}}{\partial x}-\frac{F_{1}-F_{2}}{2 H_{i}}\right) q_{i} \\
\left.+3 G_{i} q_{i}^{T}\left(\frac{\partial F_{5}}{\partial y}-\frac{F_{3}-F_{4}}{2 H_{i}}\right)^{T}\left(\frac{\partial F_{5}}{\partial y}-\frac{F_{1}-F_{2}}{2 H_{i}}\right) q_{i}\right) d v \\
T_{i}=\frac{1}{2} \rho_{i} \iiint_{V_{i}}\left(\dot{q}_{i}^{T} N^{T} N_{\dot{q}_{i}}\right. \\
+\omega\left(2 x N_{3} q_{i}+\dot{q}_{i}^{T} N_{3}^{T} N_{1} q_{i}+q_{i}^{T} N_{1}^{T} N_{3} \dot{q}_{i}^{T}-\dot{q}_{i}^{T} N_{1}^{T} N_{3} q_{i}-\dot{q}_{i}^{T} N_{3}^{T} N_{1} q_{i}\right) \\
+\omega^{2}\left(x^{2}+q_{i}^{T} N_{1}^{T} N_{1} q_{i}+q_{i}^{T} N_{1}^{T} N_{1} q_{i}\right. \\
\left.\left.\quad-x q_{i}^{T} \int_{0}^{x}\left(\frac{\partial N_{3}}{\partial x}\right)^{T}\left(\frac{\partial N_{3}}{\partial x}\right) d x q_{i}+2 x N_{1} q_{i}\right)\right) d v .
\end{gathered}
$$

\subsection{Equations of Motion}

With (2.8) the global equations of motion for the rotating plate can be obtained using Lagrange formula as follows,

$$
[M] \ddot{q}+[C] \dot{q}+[K] q=f,
$$


where

$$
\begin{gathered}
M=\iiint_{V} N^{T} N d v \\
C=2 \omega \iiint_{V}\left(N_{3}^{T} N_{1}-N_{1}^{T} N_{3}\right) d v \\
K=K_{1}-\omega^{2} \iiint_{V}\left(N_{1}^{T} N_{3}+N_{3}^{T} N_{1}\right) d v+\omega^{2} R P+\dot{\omega} \iiint_{V}\left(N_{3}^{T} N_{1}-N_{1}^{T} N_{3}\right) d v \\
f=\omega \iiint_{V} x N_{1} d v-\dot{\omega} \iiint_{V} x N_{3} d v .
\end{gathered}
$$

The integration domain $V$ is the volume of three-layer plate. As the same coupling terms between the transverse and longitudinal displacements are used, the derivation of rotating plate in this work is similar to that in [6]. It is worth noting that the proposed displacement field and derivation approach in present study are different from those in [6], where the bare rotating plate is investigated in the latter while three layer CLD structure is considered in this study.

Here the Lagrange formula is applied to each layer $\left(q_{i}\right)$ first, and then assembles the local equation of motion of each layer to form the global Equation (2.9). It can be seen that this approach can be easily extended to CLD structure with multiple layers (more than three layers), and this newly proposed model could be adopted in practical design of rotating machines such as wind turbine blades. Now, after the equation of model of three layer rotating plate is obtained, it is necessary to include the frequency dependency of viscoelastic material in the damping layer in the model.

\subsection{ADF Modeling of Frequency Dependency}

In order to include the frequency dependency of viscoelastic material, the ADF modeling approach basically introduce several additional sets of degrees of freedom (DOF) of the damping layer into the structure, and these additional sets of DOFs can be represented as $q_{i}^{a}$ where $i$ stands for $i$ th set of DOFs and the superscript $a$ stands for ADF DOFs. There could be more sets of additional ADF DOFs for different viscoelastic materials. The correlations between added ADF DOFs and (2.9) can be presented as follows [16]:

$$
\begin{gathered}
{[M] \ddot{q}+[C] \dot{q}+[K] q-\left[K_{2}^{\infty}\right] \sum q_{i}^{a}=f,} \\
\frac{C_{i}}{\Omega_{i}} K_{2}^{\infty} \dot{q}_{i}^{a}-K_{2}^{\infty} q+C_{i} K_{2}^{\infty} q_{i}^{a}=0,
\end{gathered}
$$

where $C_{i}$ and $\Omega_{i}$ are parameters for the $i$ th ADF DOFs introduced in [16]. Here, (2.11) can be combined and transformed to the first order format [17]:

$$
\left[\begin{array}{ccc}
M & 0 & 0 \\
0 & I & 0 \\
0 & 0 & \frac{C_{1}}{\Omega_{1}} K_{2}^{\infty}
\end{array}\right]\left[\begin{array}{c}
\ddot{q} \\
\dot{q} \\
\dot{q}_{1}^{a}
\end{array}\right]+\left[\begin{array}{ccc}
C & K & -K_{2}^{\infty} \\
-I & 0 & 0 \\
0 & -K_{2}^{\infty} & C_{1} K_{2}^{\infty}
\end{array}\right]\left[\begin{array}{c}
\dot{q} \\
q \\
q_{1}^{a}
\end{array}\right]=\left[\begin{array}{c}
f \\
0 \\
0
\end{array}\right] .
$$


In (2.12), there is only one set of additional ADF DOFs for simplicity, and there could be more sets of DOFs in the displacement vector $\bar{q}=\left[\begin{array}{c}\dot{q} \\ q \\ q_{1}^{a}\end{array}\right]$ which can be added similarly. Equation (2.12) can be used for solving the natural frequencies and loss factors [16], and this equation can also be transformed to state space format as:

$$
\dot{\bar{q}}=\bar{A} \bar{q}+B_{u} U+B_{m} f
$$

where $\bar{A}$ is the system matrix in state space format. $B_{u}$ and $B_{m}$ are the control matrix for externally applied control voltage and mechanical loads, respectively. The matrix $B_{u}$ is used to calculate the generated active force by an applied control signal $U$, and the matrix $B_{m}$ is a matrix that indicates an external mechanical force $f$ acting on any selected DOF. Naturally, the next section introduces how to apply a control voltage over the piezoelectric layer to add active control force into this coupled structure.

\subsection{Self-Sensing ACLD}

The piezoelectric constraining layer acts as both the sensor and the actuator. The sensed voltage is feedback through a proportional gain to apply a voltage over the constraining layer to generate active force. There are two assumptions on the ACLD in this work. First the viscoelastic layer is fully covered by the piezoelectric constraining layer, and second the applied voltage is homogenous over the whole surface of the constraining layer. In addition, proportional feedback control is adopted in this study, but more advanced control algorithms could be investigated in the future.

The constitutive equation of piezoelectric material is given in the book of Moheimani and Fleming. [18] and shown as follows:

$$
\begin{gathered}
\varepsilon_{i}=S_{i j}^{E} \sigma_{j}+d_{m i} E_{m}, \\
D_{m}=d_{m i} \sigma_{i}+\xi_{i k}^{\sigma} E_{k},
\end{gathered}
$$

where $\sigma, \varepsilon, d, E, S, \xi$, and $D$ are the stress, strain, matrix of piezoelectric constants, applied electric field, matrix of elastic compliance, permittivity matrix, and electric displacement on the surface of the constraining layer, respectively. Also, in above two equations indexes $i$, $j=1,2,3, \ldots, 6$. For these parameters, the applied electric field is calculated by applied control voltage $U$ divided by the piezoelectric layer thickness. Before applying this coupled numerical model for further studies, it is necessary to validate this new model in the next section.

\section{Validation of the Finite Element Model}

In this section, the developed finite element model is validated using data from open literature, upon which, further parametric study can be performed. In order to validate the newly proposed finite element model of ACLD rotating plate with ADF DOFs, predicted natural frequencies and loss factors by this new finite element model are compared with results from iterative modal strain energy method [15]. In this regard, we consider the parameters shown in Table 1 and the parameters of the viscoelastic material from [17]. 
Table 1: Parameters for the validation.

\begin{tabular}{lccc}
\hline Parameter & Value & Parameter & Value \\
\hline Layer 1 Thickness, $H_{1}$ & $0.003 \mathrm{~m}$ & Shear modulus 1, $G_{1}$ & $26 \mathrm{GPa}$ \\
Layer 2 Thickness, $H_{2}$ & $0.002 \mathrm{~m}$ & Shear modulus 2, $G_{2}$ & $10 \mathrm{MPa}$ \\
Layer 3 Thickness, $H_{3}$ & $0.003 \mathrm{~m}$ & Shear modulus 3, $G_{3}$ & $26 \mathrm{GPa}$ \\
Young's modulus 1, $E_{1}$ & $70 \mathrm{GPa}$ & Density 1, $\rho_{1}$ & $2700 \mathrm{~kg} / \mathrm{m}^{3}$ \\
Young's modulus 2, $E_{2}$ & $25 \mathrm{MPa}$ & Density 2, $\rho_{2}$ & $1600 \mathrm{~kg} / \mathrm{m}^{3}$ \\
Young's modulus 3, $E_{3}$ & $70 \mathrm{GPa}$ & Density 3, $\rho_{3}$ & $2700 \mathrm{~kg} / \mathrm{m}^{3}$ \\
Width, $B$ & $0.6 \mathrm{~m}$ & Length, $L$ & $0.8 \mathrm{~m}$ \\
$C_{1}$ & 1.2 & $\Omega_{1}$ & $1500 \mathrm{rad} / \mathrm{s}$ \\
\hline
\end{tabular}

1: Constraining layer, 2: damping layer, 3: base layer.
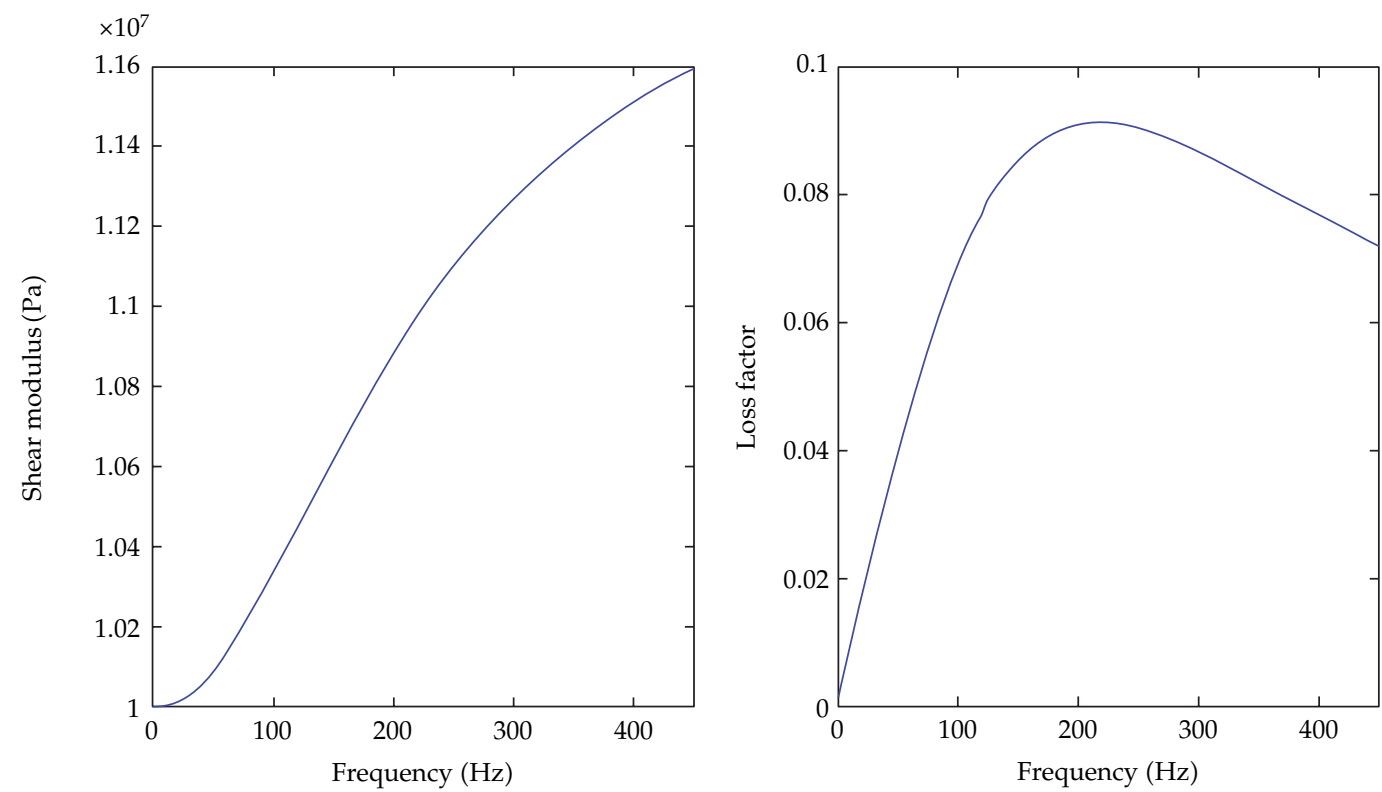

Figure 4: Frequency dependency of damping layer's shear modulus amplitude and loss factor.

Figure 4 shows with given ADF parameters how the shear modulus amplitude and loss factor of viscoelastic damping layer vary with frequency.

Table 2 shows the comparison on the first four modes. It can be seen that all of the first four modes obtained from the both approaches match pretty well to each other. In this validation, the plate is meshed with 11 nodes along both sides, and there are totally 1300 DOFs in the finite element model. It should be noted that the finite element used in validation has the same number of DOFs as models used in numerical studies which is introduced in the next section. With the validated finite element model with additional ADF DOFs, it is possible to investigate the performance of this active constrained layer damped rotating plate under different loading conditions in the next section. 
Table 2: Validation of the new plate finite element model by comparing to iterative modal strain energy (MSE) method.

\begin{tabular}{lcccccc}
\hline \multirow{2}{*}{ Modes } & \multicolumn{2}{c}{ Frequency $(\mathrm{Hz})$} & Difference & \multicolumn{2}{c}{ Loss factor } & \multicolumn{2}{c}{ Difference } \\
& New FE model & MSE method & $(\%)$ & New FE model & MSE method & $(\%)$ \\
\hline The first mode & 17.7 & 17.7 & 0 & 0.0261 & 0.0257 & 2 \\
The second mode & 32.0 & 32.1 & 0 & 0.0718 & 0.0677 & 6 \\
The third mode & 84.5 & 84.3 & 0 & 0.0892 & 0.0823 & 8 \\
The forth mode & 101.3 & 100.5 & 1 & 0.1407 & 0.1238 & 14 \\
\hline
\end{tabular}

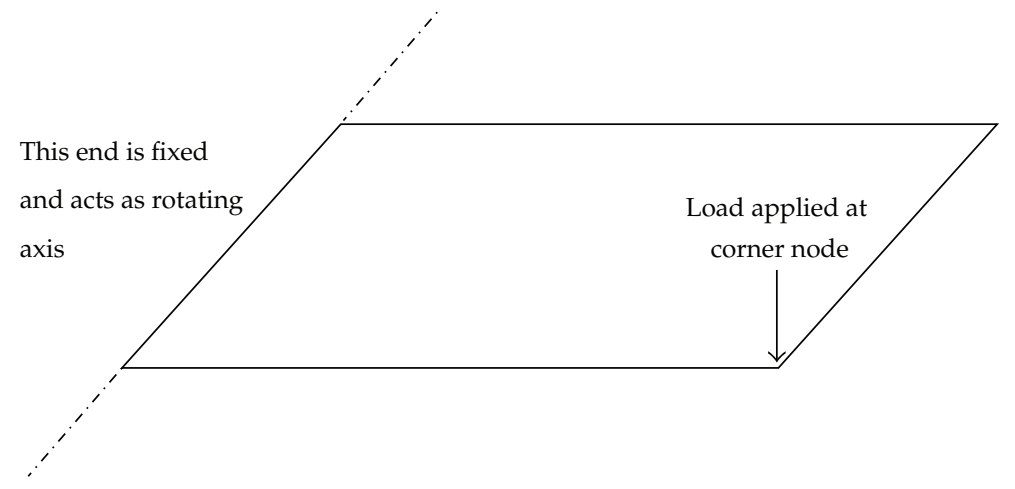

Figure 5: Location of loading and boundary conditions.

\section{Numerical Studies}

After the validation of finite element model, numerical studies are introduced in this section. The same parameters as shown in Table 1 are used in this numerical study, and two types of loading conditions are investigated: impulse $f=1 \times \delta(t)$ and unit step as $f=1$ from $t=0$. Figure 5 shows the location where these loads are applied, and it can be seen that loads are applied at the corner node of the structure. It should be noted in this numerical study that the first two modes are investigated because of their significance in vibration reduction for rotating structures such as wind turbine blades [11, 19-21].

Figures 6 to 8 show the comparisons on frequency and time responses between rotating plates with and without active control. It should be noted that in these three figures the control gain is set as 50. In Figure 6, under the impulse input, the rotating plate with active damping can damp out the vibration much faster than the one with passive damping. In Figure 7 , the active damping shows a similar performance on vibration reduction under the unit step input. Figure 8 shows the comparisons between passive and active damping on the frequency response at selected location as shown in Figure 5. Figure 8 depicts that for active damping the resonance amplitude at the first mode is drastically reduced. However, at the second mode, there is no much difference between active and passive damping. The reason is that in this study the frequency-dependent damping layer and piezoelectric constraining layer are set to fully cover the base plate, in this way bending vibration modes could be effectively suppressed while not much so for torsion modes. However, the effectiveness of the proposed method for the second mode may be very obvious, if separate damping patches are used instead of full coverage, and this configuration will be left as a future work. 


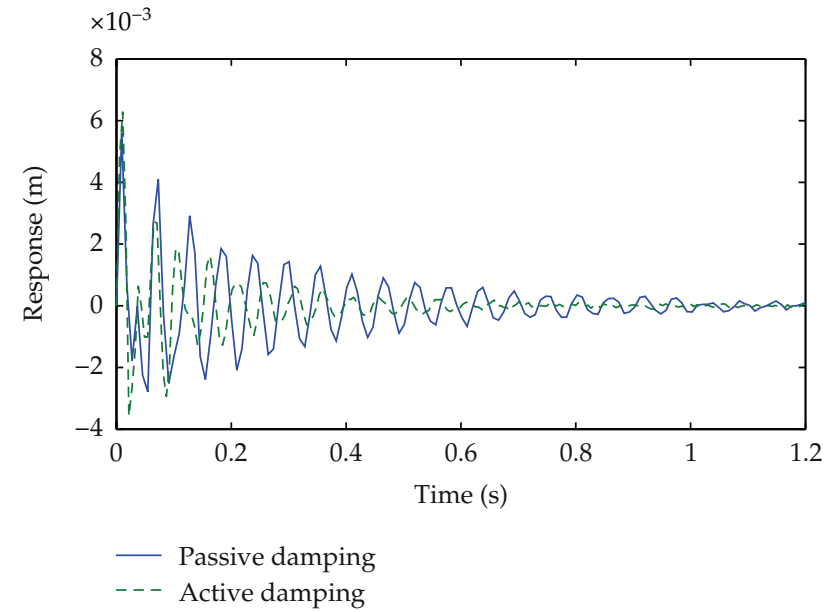

Figure 6: Comparison of time responses between active and passive damping under the impulse excitation.

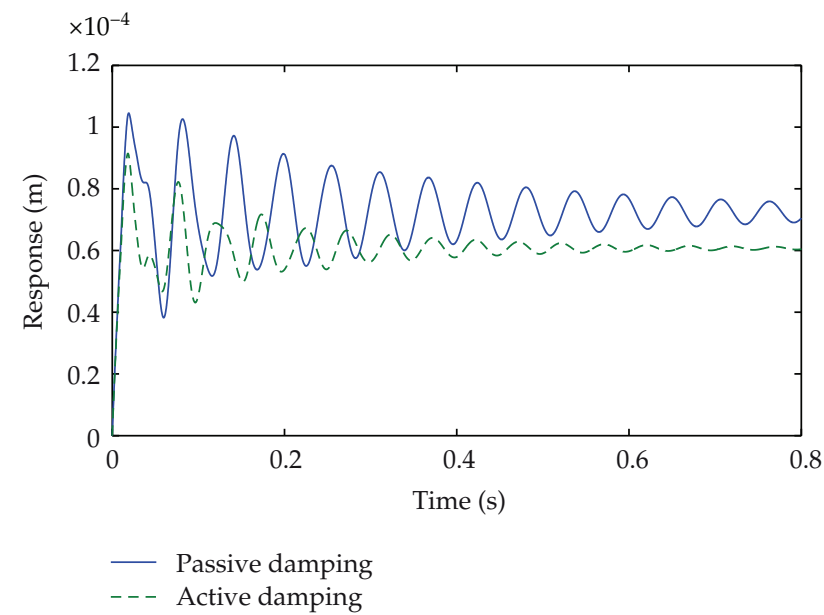

Figure 7: Comparison of time responses between active and passive damping under the unit step excitation.

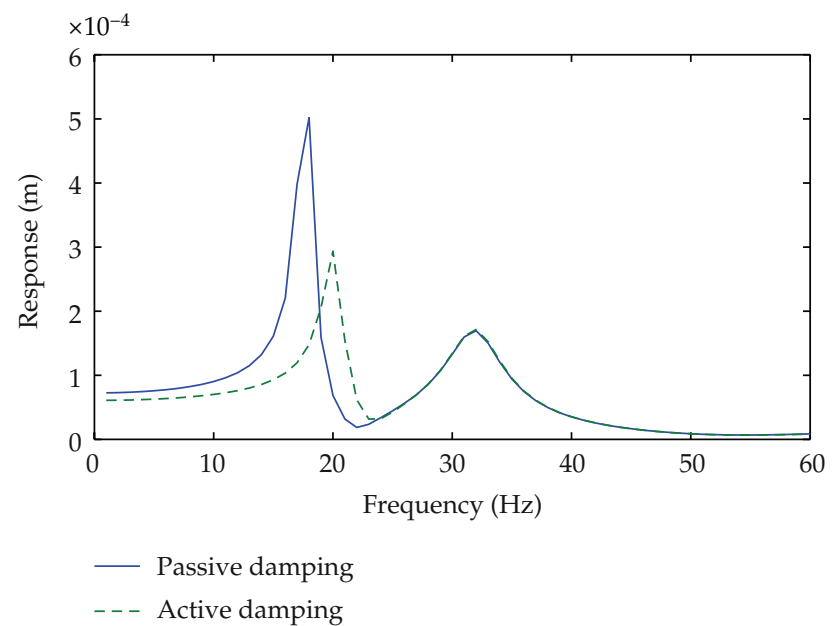

Figure 8: Comparison of frequency responses at the first two modes between active and passive damping. 


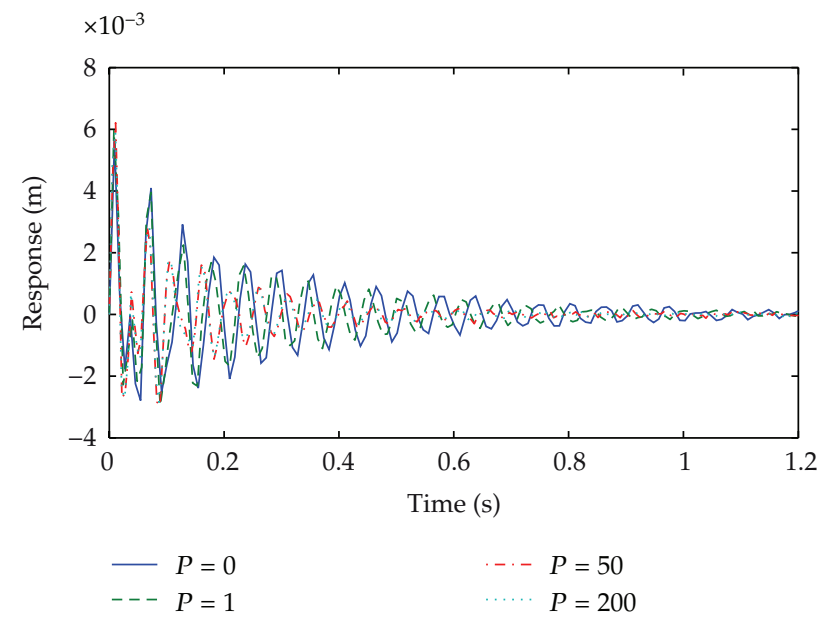

Figure 9: Comparison of time responses under the impulse excitation and different control gains $P$.

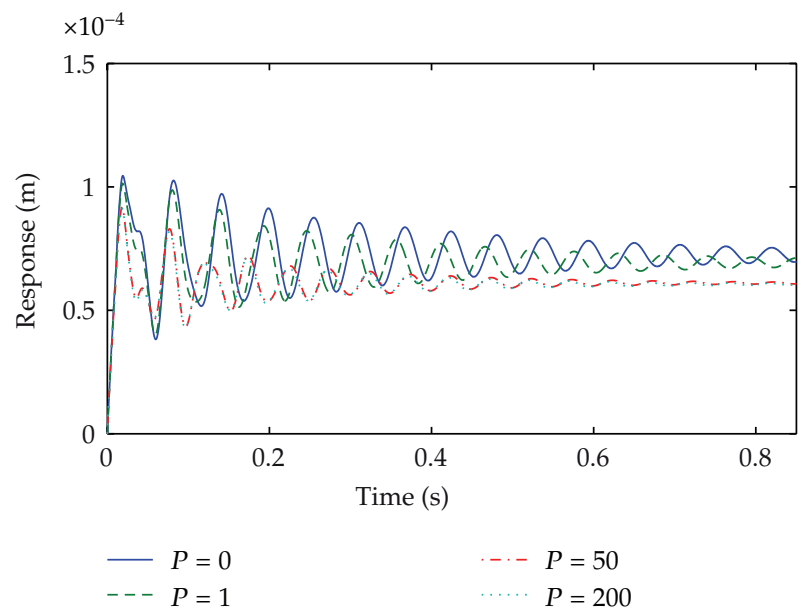

Figure 10: Comparison of time responses under the unit step excitation and different control gains $P$.

After the comparisons between the active and passive damping, it is also very interesting to check how different control gains affect the performance on vibration reduction. Figures 9, 10, and 11 show how the structure reacts to different control gains $P$. From these figures, it can be seen that as expected the higher control gain, the faster the vibration of structure is damped out. However, it can be also noticed that there is no much difference on the vibration reduction between $P=50$ and $P=200$ in both the time and frequency domains. Based on this observation, it could be concluded for this case that the performance of active damping cannot be significantly improved when the control gain reaches a certain level.

\section{Conclusion}

A new finite element model for rotating plate with coupled self-sensing piezoelectric constraining layer and frequency-dependent damping layer is developed in this work. 


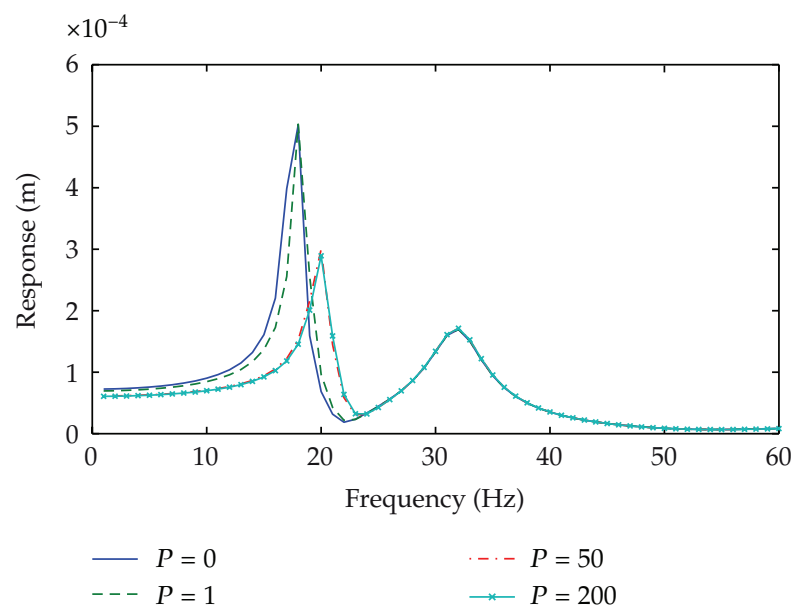

Figure 11: Comparison of frequency responses at the first two modes and different control gains $P$.

The proposed displacement field and degrees of freedom enable this finite element model to capture the shear and extension deformation in all the three layers. Also, the frequency dependency of the viscoelastic material in the damping layer is included by using ADF approach. This makes it possible to study the CLD structure in both the time and frequency domains instead of purely frequency domain analysis in the traditional complex modulus approach. This model is then validated by being compared with another proven approach on the results of natural frequencies and loss factors. In addition, numerical studies are conducted to check the performance of proposed numerical model in different loads and control conditions. The numerical results show that this newly proposed approach is effective on vibration suppression in both the time and frequency domains.

\section{References}

[1] Y. A. Khulief, "Vibration frequencies of a rotating tapered beam with end mass," Journal of Sound and Vibration, vol. 134, no. 1, pp. 87-97, 1989.

[2] H. H. Yoo and C. Pierre, "Modal characteristic of a rotating rectangular cantilever plate," Journal of Sound and Vibration, vol. 259, no. 1, pp. 81-96, 2003.

[3] J. Liu and J. Hong, "Geometric nonlinear formulation and discretization method for a rectangular plate undergoing large overall motions," Mechanics Research Communications, vol. 32, no. 5, pp. 561571, 2005.

[4] Z. Xie and X. Xue, "A new plate finite element model for rotating plate structures with constrained damping layer," Finite Elements in Analysis and Design, vol. 47, no. 5, pp. 487-495, 2011.

[5] E. H. K. Fung and D. T. W. Yau, "Vibration characteristics of a rotating flexible arm with ACLD treatment," Journal of Sound and Vibration, vol. 269, no. 1-2, pp. 165-182, 2004.

[6] L. Liu, Z. Zhang, and H. Hua, "Dynamic characteristics of rotating cantilever plates with active constrained layer damping treatments," Smart Materials and Structures, vol. 16, no. 5, pp. 1849-1856, 2007.

[7] J. J. Dosch, D. J. Inman, and E. Garcia, "Self-sensing piezoelectric actuator for collocated control," Journal of Intelligent Material Systems and Structures, vol. 3, no. 1, pp. 166-185, 1992.

[8] J. X. Gao and W. H. Liao, "Vibration analysis of simply supported beams with enhanced self-sensing active constrained layer damping treatments," Journal of Sound and Vibration, vol. 280, no. 1-2, pp. 329-357, 2005.

[9] I. Y. Shen, "A variational formulation, a work-energy relation and damping mechanisms of active constrained layer treatments," Journal of Vibration and Acoustics, vol. 119, no. 2, pp. 192-199, 1997. 
[10] J. Ro and A. Baz, "Vibration control of plates using self-sensing active constrained layer damping networks," Journal of Vibration and Control, vol. 8, no. 6, pp. 833-845, 2002.

[11] Z. Xie, P. K. Wong, and I. I. Chong, "A genetic algorithms based optimization design on self-sensing active constrained layer damped rotating plates," Journal of Intelligent Material Systems and Structures, vol. 22, no. 17, pp. 2069-2078, 2011.

[12] D. Ross, E. E. Ungar, and E. M. Kerwin, "Damping of plate flexural vibrations by means of viscoelastic laminae," in Proceedings of ASME Annual Meeting Structural Damping, pp. 49-88, New York, NY, USA, 1959.

[13] D. K. Rao, "Vibration of short sandwich beams," Journal of Sound and Vibration, vol. 52, no. 2, pp. 253-263, 1977.

[14] J. A. Zapfe and G. A. Lesieutre, "A discrete layer beam finite element for the dynamic analysis of composite sandwich beams with integral damping layers," Computers and Structures, vol. 70, no. 6, pp. 647-666, 1999.

[15] M. A. Trindade, A. Benjeddou, and R. Ohayon, "Modeling of frequency-dependent viscoelastic materials for active-passive vibration damping," Journal of Vibration and Acoustics, Transactions of the ASME, vol. 122, no. 2, pp. 169-174, 2000.

[16] M. A. Trindade, A. Benjeddou, and R. Ohayon, "Piezoelectric active vibration control of damped sandwich beams," Journal of Sound and Vibration, vol. 246, no. 4, pp. 653-677, 2001.

[17] G. A. Lesieutre and U. Lee, "A finite element for beams having segmented active constrained layers with frequency-dependent viscoelastics," Smart Materials and Structures, vol. 5, no. 5, pp. 615-627, 1996.

[18] S. O. R. Moheimani and A. J. Fleming, Piezoelectric Transducers for Vibration Control and Damping, Springer, 2006.

[19] M. E. Bechly and P. D. Clausen, "Structural design of a composite wind turbine blade using finite element analysis," Computers and Structures, vol. 63, no. 3, pp. 639-646, 1997.

[20] M. H. Hansen, K. Thomsen, P. Fuglsang, and T. Knudsen, "Two methods for estimating aeroelastic damping of operational wind turbine modes from experiments," Wind Energy, vol. 9, no. 1-2, pp. 179-191, 2006.

[21] F. Karpat, S. Ekwaro-Osire, K. Cavdar, and F. C. Babalik, "Dynamic analysis of involute spur gears with asymmetric teeth," International Journal of Mechanical Sciences, vol. 50, no. 12, pp. 1598-1610, 2008. 


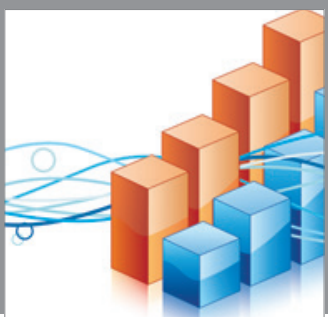

Advances in

Operations Research

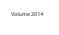

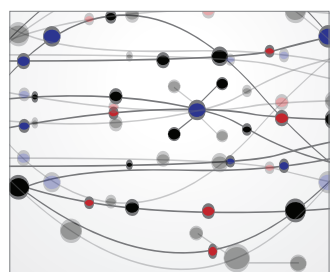

\section{The Scientific} World Journal
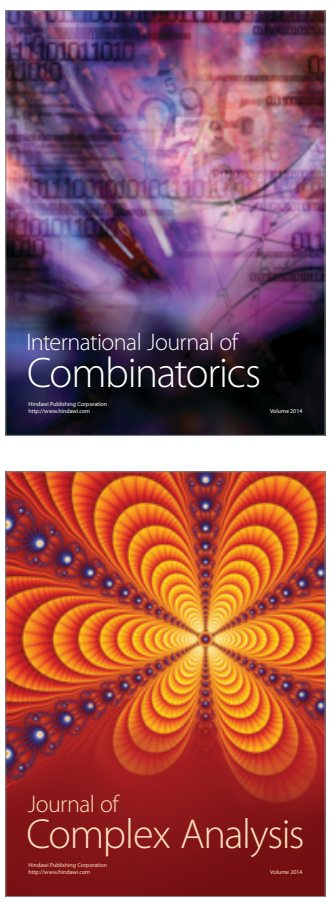

International Journal of

Mathematics and

Mathematical

Sciences
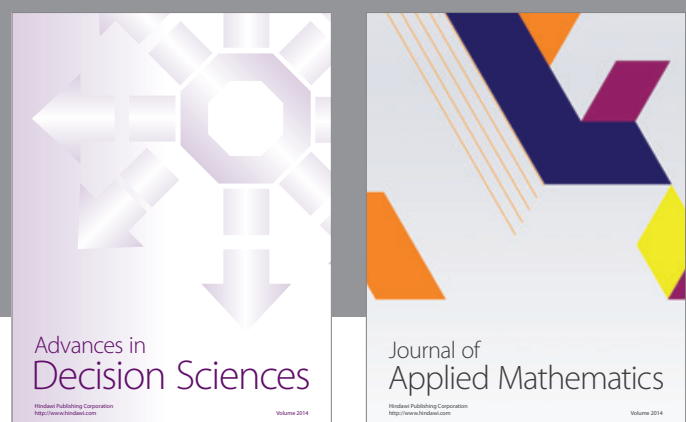

Journal of

Applied Mathematics
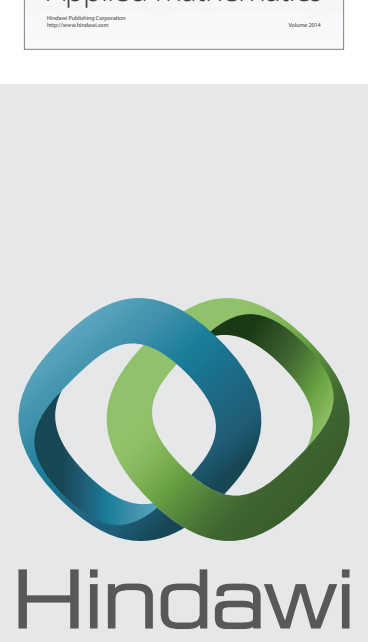

Submit your manuscripts at http://www.hindawi.com
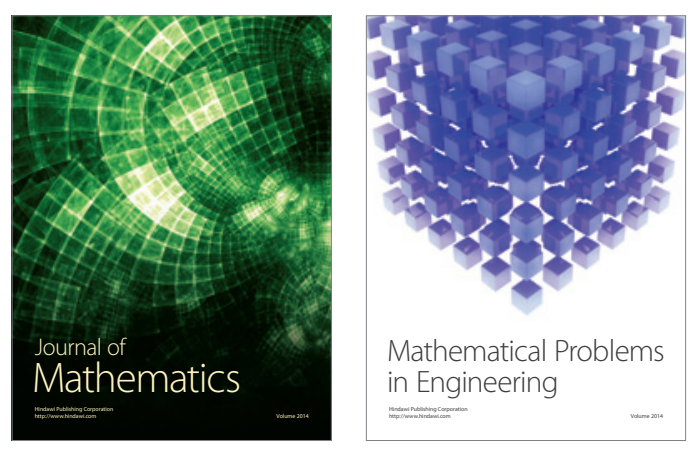

Mathematical Problems in Engineering
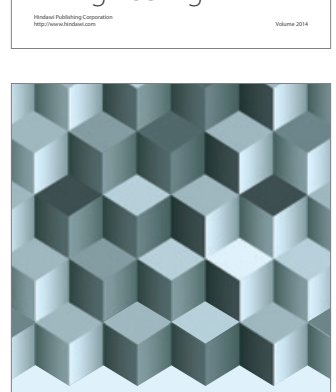

Journal of

Function Spaces
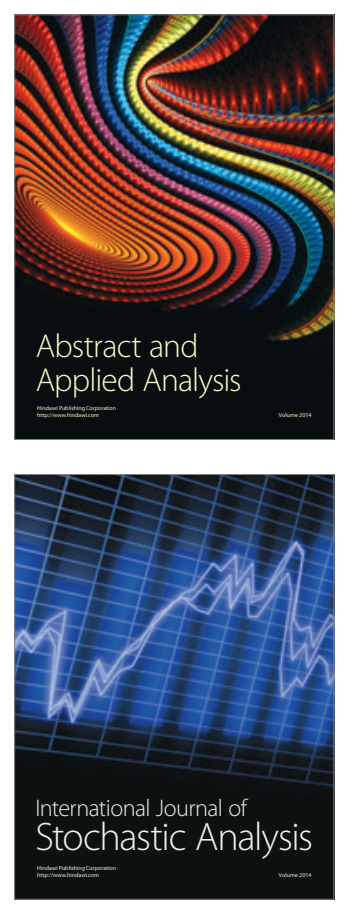

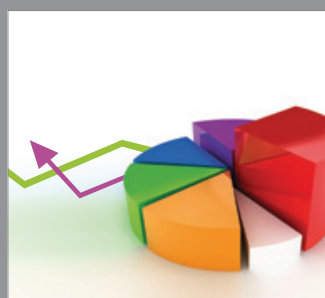

ournal of

Probability and Statistics

Promensencen
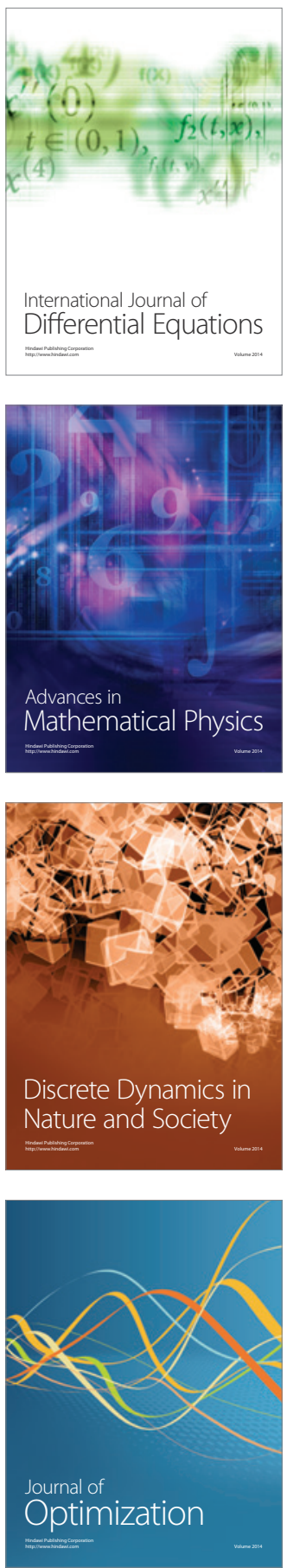